\title{
Dinámicas de apropiación social de las TIC y su impacto en las comunidades educativas.
}

Dynamics of social appropriation of ICT and their impact on educational communities.

\author{
Andrés David Hernández Mena \\ Universidad de Cartagena - Colombia \\ Andavid22@hotmail.com \\ Nuria Merlano Tous \\ Universidad de Cartagena - Colombia \\ nurycgena@yahoo.com
}




\section{Resumen}

Las Tecnologías de la Información y las Comunicaciones se han convertido en un elemento tácito para la consecución de los proyectos educativos eficientes a lo largo y ancho de la comunidad educativa a nivel nacional. Se han realizado variadas reflexiones sobre la implementación de estas en el aparato educativo, sin embargo, los estudios puntuales de casos aún son mínimos en comparación con otros tipos de análisis. Este artículo está basado en una investigación que tiene como fundamento inicial el ahondar sobre la apropiación social mediada por las TIC en dos instituciones educativas de la ciudad de Cartagena de indias, para optar al título de magister, de la maestría en educación SUE Caribe en la Universidad de Cartagena. Se observó la formación que tanto docentes como estudiantes están teniendo en estos ambientes, y para tener un mejor desarrollo en la investigación, en lo que respecta a la metodología se usó el paradigma mixto con un enfoque descriptivo, con el fin de dar mayor flexibilidad al análisis de las evidencias y tabulaciones resultantes de las visitas y análisis de fuentes.

\section{Abstract}

The Information and Communication Technologies, have become a tacit element for the achievement of efficient educational projects throughout the educational community nationwide. Several reflections have been made on the implementation of these in the educational system, however, the case studies are still minimal compared to other types of analysis. This article is based on a research that has as an initial objective to delve into the social appropriation mediated by ICT in two educational institutions of the city of Cartagena de Indias, to apply for the master's degree of the master's degree in education SUE Caribe at the University from Cartagena. We observed the training that both teachers and students are having in these environments, and to have a better development in the research, in regard to the methodology the mixed paradigm was used with a descriptive approach, in order to give greater flexibility to the analysis of the evidences and tabulations resulting from the visits and analysis of sources.

\section{Palabras Clave}

TIC, apropiación y uso de tic, buenas prácticas, innovación, mediación tecnológica.

\section{Keywords}

ICT, appropriation and use of ICT, good practices, innovation, technological mediation.

\section{Introducción}

La investigación de la cual se deriva este artículo, tuvo como finalidad analizar el proceso de apropiación social de las Tecnologías de la Información y de la Comunicación (TIC) y su impacto en la institución educativa La Libertad en el barrio El Pozón de la ciudad de Cartagena, teniendo en cuenta las prácticas educativas 
en uso de TIC que lleven a cabo los actores principales de dicha comunidad. Ahora bien, aunque estas herramientas están inmersas en la cotidianidad de esta población, y que los estudiantes no desconocen el uso de la mayoría de ellas, se hace necesario analizar de qué manera son apoyadas desde el Proyecto Educativo Institucional (P.E.I), permitiendo que se generen buenas prácticas educativas 1 (Pacheco, 2017) que premien la potencialización de los componentes que integran a la comunidad educativa para un mejor desempeño que conlleve a mejores relaciones en este ambiente educativo. El buen uso de estas herramientas fortalece la innovación en la educación, cambiando viejos modelos pedagógicos tradicionalistas, capacitando docentes que estimulen a través de la didáctica, el desarrollo de competencias tecnológicas en los estudiantes.

En este sentido, las TIC, se han convertido en una herramienta de gran valor al momento de manejar la información con fines educativos, por lo que resulta difícil poder imaginar una enseñanza sin un mínimo dominio de competencias digitales en el aula. Pero lo importante no es solo incluir estas tecnologías al ámbito educativo, sino que también desde este aspecto, permita a las comunidades desarrollarse socialmente. De tal forma, este estudio permite analizar las dinámicas de apropiación de las TIC en las comunidades educativas de las instituciones analizadas, que reflejan ciertos hábitos y que crean además en los estudiantes costumbres ajenas a su ser.

Es importante señalar, que el desarrollo tecnológico puede generar a la par una desigualdad social ya que las comunidades en situación de vulnerabilidad tienen formas diferentes de apropiación debido a "la brecha digital" entendida como "la separación entre las personas de bajos ingresos respecto a aquellos con mayores ingresos y posibilidades de acceso a la información" (Serrano, 2003, p. 34) Por lo tanto, es necesario construir una mentalidad diferente en donde se pueda transformar la realidad a través del buen uso de estos elementos tecnológicos. Debido a esto, las TIC han sido objeto de debate de diferentes entidades e instituciones. Específicamente desde el gobierno de Colombia, el ministerio de las TIC inició el Plan Vive Digital cuyo objetivo principal es impulsar la masificación y apropiación del internet con miras a tener un país más desarrollado y competitivo (MINTIC, 2012).

\section{Referentes teóricos y de Investigación}

En las últimas décadas la incorporación TIC ha sido vital, ya que en la web se encuentran formatos diferentes, contenidos digitales nuevos, gratuitos y abiertos, permitiendo la facilidad para que el usuario pueda innovar. De ahí que las políticas de tecnología en educación, apoyan a las instituciones, en la adquisición de habilidades en los estudiantes en las áreas básicas y en su vida cotidiana, para reflejar mejores resultados (Rodríguez \& Carnota, 2015).

\footnotetext{
${ }^{1}$ Las Buenas prácticas educativas en el uso mediado de las TIC son entendidas desde la postura y contribución del nacimiento de una cultura digital que favorezca al crecimiento del conocimiento mediado por las Tecnologías de la Información y las Comunicaciones.
} 
Ahora bien, dado que la actual sociedad se caracteriza por la abundante circulación de información, los individuos deberían tener el acceso de poder adquirirla, teniendo en cuenta que la calidad dependerá del propio ciudadano en seleccionar que es lo más importante, puesto que los modos de producción han cambiado, dando importancia a la mano de obra de calidad como fuente de riqueza y al manejo de la información que se potencia en las distintas economías. Esto conlleva a indagar si la sociedad también se está transformando en la escuela, siendo el sistema educativo el motor de cambio social, donde las prácticas educativas de los docentes y el sistema de formación actual son similares al siglo pasado apreciándose, deseos de mejorar (Canales \& Marques, 2007).

Actualmente, se promueve una enseñanza que incentive a la adquisición de competencias virtuales con el fin de que el estudiante sepa manejar este tipo de prácticas y así desempeñarse en la sociedad como un ciudadano capaz de ejercer en diversas áreas del conocimiento e insertarse en el campo laboral (Fundación Cartagena, 2018). Es aquí, donde las tecnologías pueden resultar útiles para alcanzar este objetivo en la sociedad del conocimiento; por ejemplo, el manejo de foros, correos electrónicos, chats, videoconferencias, plataformas educacionales, páginas web, bases de datos, entre otras, pueden ser de gran utilidad permitiendo el dinamismo en las clases.

Sin embargo, estas tecnologías a su vez pueden presentar desventajas si no se les da un uso adecuado. Algunas desventajas son la falta de conocimiento en el manejo de estas herramientas, costo de los equipos, la insuficiencia de éstos, la dependencia y abuso de la tecnología, y la falta de interés de organizar las asignaturas de acuerdo a los materiales tecnológicos de una institución para poder dar paso a la innovación.

En algunos estudios como el de Condie y Munro (2007), se observa que en los lugares donde las TIC se constituyen en parte integral del proceso educativo, hay mayores evidencias de impacto positivo en el aprendizaje y el desempeño de los estudiantes. Estas observaciones, orientan a poner en prácticas otras estrategias de aprendizajes que podrían facilitar el interés de los estudiantes y así facilitarle al docente la construcción de los conceptos. Sin embargo, todo depende también de las capacidades, actitudes y creencias pedagógicas de los docentes, ya que algunos no tienen la formación en el uso de tecnologías, y esto puede causar confusión o fracaso en la interpretación del aprendizaje.

Por otro lado, Barón y Gómez (2002) al analizar la trayectoria de las políticas públicas en torno a TIC en Colombia, mencionan que es importante destacar que a pesar de todos los esfuerzos realizados las políticas públicas siguen teniendo un enfoque meramente instrumental que solo se preocupa por la difusión y el acceso y olvida aspectos como la integración, uso e innovación de las TIC en la vida de las distintas comunidades. Es aquí que proponen fortalecer la investigación y masificación de la información en cuanto a experiencias de uso, y 
también promover la adopción y desarrollo de programas de TIC. De igual forma, Polanco (2012) sostiene que el funcionamiento de una política pública en torno a las TIC depende de la voluntad para implementarla, en destinar recursos para este campo, en el compromiso de las directivas de las instituciones educativas, en comprometer a la comunidad en el uso de las TIC, y capacitar a los docentes en el manejo de estas herramientas.

De acuerdo con esto, Said-Hung, Iriarte, Jabba, Barreto, Ballesteros, Vergara y Ordóñez (2015) señalan que más que promover un carácter instrumental de las TIC, se debe transformar esta situación hacia la búsqueda de la consolidación del perfil docente y el papel que deben cumplir en los procesos de aprendizaje, fortaleciendo los procesos de formación hacia el aprovechamiento de los recursos tecnológicos. En este sentido, la escuela como motor de innovación debe estar dispuesta a iniciar una gestión educativa, implementado estrategias pedagógicas mediadas por TIC, y promoviendo la utilizando de contenidos digitales. Para ello, se debe promover la formación de los docentes en el uso responsable y la apropiación de TIC como estrategia para fortalecer el desarrollo de competencias en los estudiantes. (Pacheco, 2017, p. 87).

\section{Metodología}

En este estudio de carácter mixto, se tuvo en cuenta el contexto y las condiciones de la población, por lo que fue necesario hacer uso de una metodología flexible pero no menos rigurosa como forma de interpretar la realidad de los participantes. En este sentido, el enfoque mixto provee herramientas de carácter cualitativo y cuantitativo para obtener evidencias a través de entrevistas, planes de estudios, tabulaciones, análisis estadísticos, capacitaciones tecnológicas docentes y experiencias significativas que desde la apropiación social son de rescatar para las intenciones finales de este estudio.

Con relación a esto, Creswell (2008), sostiene que la investigación mixta permite amalgamar, en un solo estudio, prácticas metodológicas cuantitativas y cualitativas, con el propósito de que exista una amplia y mayor comprensión acerca del objeto de estudio. Aspecto que, en el caso de los diseños mixtos, puede ser una fuente de explicación a su surgimiento y al reiterado uso en ciencias educacionales que tienen relación directa con los comportamientos sociales.

Por otra parte, se trabajó desde un enfoque descriptivo, de tipo documental donde a partir del estudio y análisis de referencias bibliográficas, se permitió plantear consideraciones en cuanto a uso de las TIC e innovación tecnológica en educación, problemas de la utilización de Internet por parte de niños y adolescentes, presentación de una propuesta de cursos de formación a docentes en ejercicio para que puedan utilizar eficazmente estas herramientas tecnológicas en su quehacer didáctico.

Además, se analizaron las actitudes de los diferentes actores en estas comunidades educativas de acuerdo al manejo de tecnologías, las relaciones entre ellos, y la comprensión de las prácticas educativas en donde hay incidencia de ese tipo de herramientas. Todo esto apoyado por los datos cuantitativos 
derivados de las encuestas aplicadas con los cuales se pudieron determinar los hallazgos en cuanto a impacto o falencias de los procesos educativos.

Con relación a los instrumentos utilizados, se siguieron las recomendaciones de Pacheco (2017), al aplicar una encuesta para docentes y otra para estudiantes con miras a recopilar información en diferentes aspectos relacionados con la formación, uso de recursos y herramientas TIC, competencias, cambios en la práctica educativa y pedagógica, necesidades, entre otros. La muestra estuvo conformada por docentes y estudiantes de la I.E La Libertad en la ciudad de Cartagena; donde se tomaron 6 docentes y 30 estudiantes de grado $11^{\circ}$ para la realización de las encuestas, además de trabajo de observación.

A partir de los instrumentos definidos a utilizar, teniendo en cuenta su validación por los autores de los mismos, se procedió a su adaptación. Para comprobar su funcionamiento, fidelidad y validez de las respuestas y resultados, se realizó un cuestionario piloto para conocer el juicio de 5 docentes de distintas asignaturas y 5 estudiantes que proporcionaron un concepto aceptable y permitieron rectificar algunos aspectos antes de llevar el instrumento a la práctica. Una vez se obtuvieron los resultados, estos fueron sometidos a la prueba Alpha Cronbach para obtener su grado de confiabilidad (Celina \& Campo, 2005).

Las etapas que se llevaron a cabo fueron las siguientes:

- PRIMERA ETAPA: Se hizo la reflexión y planificación, en donde se identificó el problema, y se desarrollaron las primeras revisiones documentales acerca del interrogante. Luego se seleccionó el escenario de investigación, las estrategias para abordarlo y se redefinió el problema.

- SEGUNDA ETAPA: Se realiza la recogida de información en campo, se seleccionó la muestra y se aplicaron las técnicas e instrumentos diseñados para tal fin.

- TERCERA ETAPA: Se llevó a cabo el análisis de la información a través de métodos cuantitativos (análisis estadístico, cálculo de porcentajes, y correlaciones), y cualitativos (reducción de datos, transformación de porcentajes en conclusiones) que permitieran la elaboración del informe.

Por otra parte, en el estudió se definieron tres categorías: Didáctica y apropiación social de TIC por los docentes, Aprendizaje por el uso de TIC en los estudiantes y Cambios institucionales en el P.E.I, las cuales orientaron la fase metodológica y la obtención de resultados de la investigación.

La primera categoría, se centró en la necesidad de revisar y reformular la utilización de las TIC en la educación, de manera que ayuden a establecer mejores formas de actuar con los medios de enseñanza, permitiendo una articulación entre docentes, medios de enseñanza y preparación pedagógica y metodológica.

La segunda categoría, surgió del reconocimiento del avance, desarrollo e impacto del uso de TIC, las cuales han ido permeando en los diferentes procesos 
de aprendizaje en los estudiantes de la sociedad moderna. Castro (2017), menciona que de acuerdo a las exigencias de la sociedad actual, se hace necesaria la formación en competencias digitales en los estudiantes y que les ayude no solo a ser productivos sino también efectivos. Estas competencias son la creatividad, la innovación, la comunicación, la colaboración, la investigación, el manejo de la información, el pensamiento crítico, la solución de problemas, la toma de decisiones, conocimiento de los conceptos de ciudadanía digital y funcionamiento de las TIC.

La tercera categoría, se relaciona con la carta de navegación de las instituciones educativas, y la necesidad latente de vincular a dicha normatividad una planificación de actividades y procesos de enseñanza que tenga presente a las tecnologías como recurso didáctico. En Colombia el Plan Decenal de Educación 2016 - 2026 centra uno de sus desafíos a "impulsar el uso pertinente, pedagógico y generalizado de las nuevas y diversas tecnologías para apoyar la enseñanza, la construcción de conocimiento, el aprendizaje, la investigación y la innovación, fortaleciendo el desarrollo para la vida. Dentro de este, se encuentra que uno de sus lineamientos específicos es el que busca "Incentivar el uso de las TIC en la práctica docente de forma pertinente en los procesos de planeación curricular, enseñanza y seguimiento del aprendizaje de los estudiantes" MEN (2017).

\section{Resultados y Discusión}

\section{Análisis de la Apropiación de TIC en los Docentes de la Institución Educativa La Libertad.}

Como se mencionó anteriormente, se seleccionaron 6 maestros de la institución educativa, a los cuales se les aplicaron las encuestas relacionadas con aspectos como la formación, percepción acerca de las TIC y la innovación pedagógica. También se elaboraron entrevistas con preguntas que profundizaban sobre aspectos específicos de la encuesta, con fin de recopilar la máxima información de los docentes.

Con respecto a la Experiencia sistematizada en el uso educativo de las TIC desde su Institución Educativa, la mayoría de docentes manifestaron tener experiencia, mientras la minoría dicen no tenerla; esto indica que en mayor porcentaje los docentes encuestados conocen de las TIC. En cuanto a la formación y percepción en TIC, los docentes refieren en su mayoría, haber asistido a cursos de formación, seminarios, conferencias, estudios de posgrado, cursos de corta duración o diplomado. Algunos de estos cursos fueron realizados por convocatoria de la Secretaria de Educación, por llamado de la Institución Educativa o por iniciativa propia de los docentes.

Al evaluar el desempeño y su liderazgo, el mayor porcentaje de respuestas fue negativo, donde no exploran ni discuten los atributos de las comunidades de aprendizaje digitales para mejorar la labor de enseñanza con sus estudiantes, ni 
la realización de prácticas profesionales prometedoras en apoyar el aprendizaje de éstos. Por tanto, las respuestas afirmativas de menor porcentaje se encaminaron en la participación a desarrollar actividades orientadas a adoptar las TIC en la institución educativa y la comunidad educativa donde se trabaja. EI desempeño relacionado con la utilización de TIC a través de email, chat foros, mensajes de texto es medio al igual que la participación en comunidades virtuales y redes de aprendizaje. Entre las actividades de desempeño menos utilizadas, están la búsqueda y selección de información utilizando herramientas TIC y fuentes como buscadores, bibliotecas virtuales y repositorios.

Son actividades comunes el traslado de equipos disponibles de la institución educativa al aula de clase, llevar sus equipos al aula de clase y utilizar los equipos que disponen los estudiantes como celulares y tabletas. De acuerdo a las opciones que se ajustan al perfil del docente en su desempeño en la promoción de la ciudadanía digital, los docentes responden, a facilitar el acceso equitativo de herramientas TIC en sus estudiantes, orientadas a las diversas necesidades que necesitan, como el aprovechamiento de los recursos para la promoción de diversos estilos de aprendizaje para los estudiantes y promover el uso adecuado y la aplicación ética de éstos. Los tipos de barreras que impiden el acceso a los equipos a los docentes en mayor porcentaje están relacionados con las variables que indican la inexistencia de los equipos y que los pocos que tienen se encuentran dañados.

Los equipos utilizados frecuentemente con fines educativos son: EI PC portátil, equipo de sonido, aplicaciones móviles, Smartphone. De acuerdo a esto, los docente mencionan que aprovechan la tecnología para la práctica de situaciones de la vida real, como la comunicación y colaboración con otros miembros de la comunidad educativa para compartir información y establecer relaciones con estos, es decir, mayoritariamente prueban y usan variedad de TIC para comunicarse e intercambiar información con otras personas de otras partes del mundo y mirar la efectividad con fuentes de información que sirven de apoyo a la investigación y aprendizaje propio y de los estudiantes y miembros de la comunidad educativa.

\section{¿Qué usan, qué hacen y que aprenden con TIC los estudiantes de la Institución Educativa La Libertad?}

Para el estudio se seleccionaron 30 estudiantes de educación media de la institución educativa, los cuales manifestaron con relación a la apropiación y uso de dispositivos y recursos TIC que el televisor, el chat, y el internet, son los recursos más utilizados en la semana. En un rango de menos de una vez por semana se encontró que Los Smartphone, los buscadores, el equipo de sonido, el alojamiento de video en youtube, y el uso de portátil se encuentran en esa variable de uso. Mientras que, en un rango de uso ocasional está el portátil, el computador de escritorio, la Tablet, el DVD, la cámara fotográfica, el celular básico, la impresora y wikis. 
En relación a los lugares donde los estudiantes usan los dispositivos y recursos TIC, se encontró que, en el colegio, específicamente en la sala de informática usan internet, el blog, el tablero digital, el computador de escritorio, las cámaras digitales, las tabletas, equipo de sonido, y el Smartphone. En el salón de clase utilizan aplicaciones móviles, equipo de sonido, impresoras. En el cibercafé utilizan televisor y buscadores. Los lugares donde no utilizan dispositivos y recursos TIC son: casa de familiares, casa de compañeros de estudios o amigos y en sus casas.

De acuerdo al interés de los estudiantes los recursos fueron clasificados de la siguiente forma: el internet, es mayoritariamente usado para buscar información sobre temas de interés. El computador de escritorio, para compartir e intercambiar información. Aplicaciones como skype, impresora, redes sociales, y blog son usados para subir o descargar contenidos. El chat, el portátil y el kit multimedia son usados para juegos online o distraerse. Las wikis, las aplicaciones móviles, los foros virtuales, el portátil, el DVD, la filmadora, la cámara fotográfica, el equipo de sonido, el smartphone, el escáner, celular básico y la impresora, son utilizados para trabajar y comunicarse con los compañeros y profesores de clase. El televisor, y los buscadores para hacer tareas de clase.

Las dificultades que presentan los estudiantes para hacer uso de recursos y dispositivos TIC en su colegio, tienen relación con la posibilidad de utilizar los dispositivos fuera de la clase. En segunda instancia, se encuentra que hay pocos dispositivos para el número de estudiantes de los grupos, y también se evidencia una gran cantidad de equipos dañados. Con base a esto se cuestionó que tipo de problemas dificultan el uso de los recursos TIC en la institución, teniendo como resultado que la barrera que más identifican los estudiantes es la relacionada con el uso de equipos fuera de la clase. Este problema, es seguido por la variable que señala la poca cantidad de equipo para la población estudiantil. Otros limitantes no menos importantes son los relacionados con los equipos que están dañados, y que siempre se requiere autorización para poder utilizar estos recursos.

La frecuencia de uso de los estudiantes con las TIC se caracteriza por el interés en intercambiar información con sus compañeros para realizar presentaciones y trabajos, publicar fotos, videos, realizar prácticas de foros, utilización de wikis, blogs, software educativo de matemática, inglés, ciencias sociales y comunicarse con el profesor. Las materias que más frecuentan el uso de TIC en la escuela son tecnología, español e inglés y las que menor uso realizan con TIC son las ciencias, educación artística, educación física, ética, religión y matemáticas. Las actividades que realizan los estudiantes ocasionalmente en las comunidades virtuales se relacionan con los trabajos, investigaciones y la búsqueda de fuentes de información, así como el uso de plataformas de interacción con profesores y compañeros de grupo.

\section{El Proyecto Educativo Institucional en la I.E La Libertad.}

Desde el horizonte institucional, se hacen alusión al uso de TIC, ya que resaltan importancia de proyectar una educación que posibilite la formación 
integral de ciudadanos a través de la inclusión de las tecnologías en el currículo. Así mismo, resaltan el conocimiento científico, desde los fundamentos epistemológicos del P.E.I como estrategia para que el estudiante pueda interpretar la realidad que rodea a la institución. Sin embargo, cuando se habla de pedagogía, se dejan de lado las TIC y se centran en utilizar el modelo constructivista, sin mencionar si se apoyaran en esas herramientas para lograr su objetivo.

A pesar de todo, no se desconoce los grandes avances que pueden lograrse con el uso de las tecnologías. Se menciona en el P.E.I que la educación actual debe garantizar la formación de un ciudadano competente que pueda aprovechar los avances tecnológicos que se le ofrece. En el manual de convivencia, se encuentra establecido en la sección de derechos de los estudiantes, que: "Es derecho de los estudiantes, recibir educación de calidad de acuerdo a los avances científicos y tecnológicos..." y menciona también "utilizar racionalmente los espacios e instalaciones como la sala de sistemas, canchas, laboratorios, según los horarios y reglamentos dispuestos". Con esto se hace visible una total disposición a ofrecer abiertamente las instalaciones y recursos disponibles para que los estudiantes puedan hacer sus prácticas en TIC. En el punto de obligaciones especiales de la I.E, menciona que "se debe garantizar la utilización de las TIC y espacios culturales como la biblioteca" apartado que llama la atención, debido a que la biblioteca es el lugar menos dotado de recursos y en el momento de la investigación, este sitio era utilizado como lugar de archivo y resguardo de implementos.

Por otra parte, desde el documento la interdisciplinariedad se ha visto como una preocupación, pero también como una necesidad que impone los retos de la sociedad actual. Es entendible entonces, como las instituciones educativas, a través de sus funciones deben procurar incentivar la interdisciplinariedad y promover un pensamiento crítico analítico y de igual forma una ciudadanía activa que contribuya al establecimiento de prácticas que ayuden a mejorar la calidad de vida de las personas. Llevarla a la práctica ayuda a evidenciar las conexiones entre los diferentes conocimientos, asignaturas, disciplinas, y con ella se puede adquirir una concepción científica de la realidad, permitiendo demostrar que los fenómenos no existen por separado, sino que se encuentran interrelacionados favoreciendo a tener una visión más amplia de las cosas.

A pesar de todo, es un hecho que en lo educativo, en la institución se muestra poca aplicación o puesta en práctica de lo planteado en el P.E.I, puesto que en la mayoría de los casos se carece de herramientas (teórico - metodológicas) para llevar a cabo el camino hacia la interdisciplinariedad. Esto es visible desde el trabajo de las diferentes disciplinas de las asignaturas y hasta de cada uno de los temas a abordar. No obstante, en la institución son conscientes que la interdisciplinariedad en la educación se constituye como una necesidad ante los retos actuales, y por lo tanto, todo lo que se haga para alcanzar su perfeccionamiento es válido para la formación de un hombre capacitado que incida en la sociedad; así mismo, se vislumbra la interdisciplinariedad como un factor que ayuda a la optimización del proceso formativo y alcanzar una educación reflejada en el modo de actuación de los educandos. Por lo que el trabajo 
metodológico desde cada instancia organizacional de la educación, constituyen la base que garantiza la apropiación y utilización de las TIC como medios de enseñanza en el proceso educativo.

De ahí, que en la institución educativa la Libertad, en el año 2016 se les hizo entrega de unas tabletas a docentes y estudiantes para que hicieran un uso de las TIC, como iniciativa para propiciar la apropiación tecnológica. Sin embargo, no se ha establecido criterios claros para llevar a cabo proyectos de interconectividad dentro de la institución que les ofrezcan a maestros y educandos un acceso permanente a la red, lo que se constituye en un obstáculo claro para llevar a cabo una apropiación real y didáctica de los medios tecnológicos.

Por otro lado, desde el hogar y de manera extracurricular, con estos dispositivos puede existir una interconectividad entre docentes y estudiantes si se dan las condiciones. Sin embargo, hay que tener en cuenta que no todos los estudiantes de la institución educativa la Libertad tienen acceso a internet y planes de datos, por lo cual esta intención no podría llevarse a cabo. Se puede inferir que es apremiante la vinculación de los aparatos administrativos, docentes y estudiantiles a este tipo de iniciativas que lo que buscan es reducir a su más mínima expresión la desigualdad en cuanto a la preparación de los educandos. Por este motivo, la apropiación tecnológica en ambientes polivalentes permite la interacción y el derribamiento de las barreras de información, que en ocasiones por falta de recursos en unos $u$ otros lugares se manifiesta en ausencia de oportunidades y limitaciones a datos e interactividad.

\section{Discusión}

Los resultados demuestran, que si bien los docentes realizan cursos de formación en TIC, su interacción con las TIC no deja de ser meramente instrumental, considerando las tecnologías como herramientas para facilitar y continuar sus prácticas de carácter tradicionalista. Esta situación lleva a replantear el postulado de la investigación de Said-Hung (2015) "sin capacitación docente no se elimina el carácter instrumental que se les da a las TIC". De igual forma lleva a evidenciar lo propuesto por Barón y Gómez (2002) en donde dice que "Ias TIC son instrumentales y en nuestro país se preocupan solo de difundir y generar acceso".

De acuerdo con esto, no se trata solamente que los docentes tengan la oportunidad de formarse en tecnologías, sino que lo aprendido se lleve a la práctica, y que además los directivos se preocupen por garantizar un plan de acción en donde se le dé importancia a la inclusión de TIC desde el currículo. Esto guarda relación con el planteamiento de Pacheco (2017) quien considera que "solo hay innovación educativa cuando hay gestión de inclusión de contenidos digitales y prácticas TIC en el currículo y formando a los docentes". Así pues, que es función de las directivas del plantel educativo, preocuparse de que sus docentes que cuenten con capacitación en el manejo y aplicación de tecnologías 
en la educación, las lleven a la práctica y generen impactos positivos en los aprendizajes de los estudiantes.

Con respecto a los estudiantes, se encontró que la población utiliza mayormente algún tipo de tecnología únicamente cuando están en la institución educativa. Esto está ligado a un factor como el contexto, ya que la comunidad pertenece al barrio El Pozón en la ciudad de Cartagena, un sitio con altas cifras en fenómenos como pobreza, economía informal, familias disfuncionales, y en donde el uso de las tecnologías casi tiene la connotación de ser un lujo. La institución educativa se presenta como la oportunidad de tener contacto con las TIC, en donde pueden acceder a internet, aunque los equipos sean insuficientes y existan barreras que dificultan la práctica con estas herramientas. Al respecto, es necesario tener en cuenta a Canales y Marques (2007) al manifestar que "la sociedad se está transformando desde la escuela, su motor de cambio social en donde las practicas docentes y la formación lleva a desear mejorar". De ahí, que el rol trasformador de la escuela es de gran importancia para generar procesos de uso y apropiación de la tecnología de manera responsable y estructurada.

En cuanto al P.E.I, este menciona la importancia de la inclusión de TIC en los ámbitos de formación de la I.E, el currículo, y en aspectos de dotación de tecnologías, pero se evidencian vacíos como la articulación de lo estipulado en el P.E.I con lo que se hace en la práctica. Muestra de esto, es la dotación de tabletas a docentes y estudiantes en 2016, pero no se elaboró una guía curricular para definir su uso de acuerdo a los objetivos que se proponen alcanzar a nivel institucional en la gestión académica.

\section{Conclusiones}

A partir de los resultados y discusión de este estudio, es posible considerar que los docentes no son indiferentes a la formación y actualización en TIC, incluso, aun cuando la institución educativa no hace gestión para la realización de talleres y cursos, optan por hacerlos por cuenta propia. Esto ha llevado a que los docentes acepten la inclusión de algún tipo de tecnología en sus prácticas demostrando la manifestación de cierto grado de importancia. A pesar de lo anterior, se mantienen vigentes formas didácticas tradicionales en las actividades docentes. La clase magistral predomina, lo cual dificulta la articulación de las practicas con la interdisciplinariedad, el uso de redes o web 2.0, dando paso así al desaprovechamiento de un trabajo colaborativo que se puede generar gracias a esas tecnologías. Esto es apoyado en cierta forma por las directivas institucionales, ya que a pesar de la existencia de algún tipo de tecnología, y aunque se disminuyan barreras como el acceso y tiempo de uso, el docente opta por continuar en lo tradicional.

Los estudiantes hacen uso diario de la tecnología gracias al acceso a Smartphone e internet. Este último se facilita por la conexión en sus escuelas o la compra de paquetes de datos que son comunes entre los operadores de telefonía nacional. Son hábiles en el intercambio de información, y algunos utilizan aplicaciones que les permitan desarrollar sus actividades escolares. Sin embargo, el uso de las tecnologías anteriormente mencionadas, se ve obstaculizado en la 
escuela debido a la autorización que proviene de administrativos y docentes para poder utilizarlas fuera de clase.

El análisis del PEI permitió observar, que aunque se mencionan los fines de la educación acorde a las TIC en estos documentos, y aunque hay dotación de recursos, no hay una integración de esos elementos con la misión, visión, y la malla curricular. Por lo tanto, a pesar de que los docentes tengan formación en tecnologías, sus prácticas seguirán siendo guiadas por metodologías tradicionales. Las directivas de la institución no incentivan continuamente la promoción de programas y planes que apoyen la dotación de recursos TIC, pero sobre todo, no se evidencia una vinculación apropiada al currículo, por lo que las practicas mediadas por TIC difícilmente podrán ayudar a alcanzar los objetivos que se proponen en el PEl.

No obstante, las TIC son herramientas que lideran las dinámicas educativas y sociales, poco a poco se van haciendo visibles en la cotidianidad de estas comunidades, permeando las practicas que ejercen estas personas. En este sentido, los recursos tecnológicos dotan de enormes posibilidades a docentes y estudiantes, al proveer diversas fuentes de información y posibilitar distintos escenarios de interacción para promover aprendizajes pertinentes y significativos.

\section{Referencias}

Barón F, L y Gómez, R. Las políticas públicas sobre TIC en Colombia: de la infraestructura a la apropiación social. EN: Más que teclas y pantallas. Acceso público a TIC en Colombia 1 ed. Universidad Icesi. Facultad de Derecho y Ciencias Sociales, Cali, 2014.

Belloch, C. (2012) Las Tecnologías de la Información y Comunicación en el aprendizaje. Material docente [on-line]. Departamento de Métodos de Investigación y Diagnóstico en Educación. Universidad de Valencia.

Canales, R., \& Marqués, P. (2007). Factores de buenas prácticas educativas con apoyo de las TIC. Análisis de su presencia en tres centros educativos. En Revista Educar, (39), pp. 115-133.

Castro, J. (2017). Impactos educativos por la incorporación de las herramientas tecnológicas en los procesos de enseñanza - aprendizaje en cuatro instituciones educativas oficiales del distrito de Cartagena de indias. Tesis para optar al título de magister en educación. SUE Caribe Universidad de Cartagena.

Celina, H; Campo, A. (2005). Aproximación al uso del coeficiente alfa de Cronbach. Revista Colombiana de Psiquiatría, vol. XXXIV / No. 4.

Condie, R., \& Munro, R. (2007). The impact of ICT in schools: A landscape review. Strathclyde: University of Strathclyde.

Cortés, S. M. (2017). Uso de las TIC en la práctica pedagógica. En: Revista TIA, 5(1), pp. 46-56.

Creswell, J. (2008). Mixed Methods Research: State of the Art. [Power Point Presentation]. University of Michigan. Recuperado de sitemaker.umich.edu/creswell.workshop/files/ creswell_lecture_slides.ppt 
Escorcia-Oyola L, de Triviño C. (2018) Tendencias de uso de las TIC en el contexto escolar a partir de las experiencias de los docentes. Educación Y Educadores [serial online]. January 2015;18 (1):137-152. Available from: Fuente Académica Premier, Ipswich, MA. Accessed January 8,

Fundación Cartagena. 2018. Cómo vamos. Disponible en http://www.cartagenacomovamos.org/como-vamos-en/cartagena-2/. Consultado el 1 de enero de 2018.

Institución Educativa Nuestra Señora de la Consolata. (2012). Manual de Convivencia de Institución Educativa Nuestra Señora de la Consolata. Cartagena.

MEN. (2017). Plan Nacional Decenal de Educación 2016 - 2026. Ministerio de Educación Nacional. ISBN: 978-958-5443-46-4. Bogotá

Pacheco, A. (2017). Buenas Prácticas en uso de TIC en las Escuelas Innovadoras del Caribe Colombiano. Ediciones Tecnológica de Bolívar. Cartagena.

Polanco, C. (2011) Evaluación de los impactos de las políticas públicas en la apropiación social de las TIC, caso Medellín - Colombia. EN: Revista AHCIET: revista de telecomunicaciones, ISSN-e 0213-1226, No. 125.

Institución Educativa La Libertad. (2012). Proyecto educativo institucional. Institución educativa La Libertad. Cartagena.

Institución Educativa Nuestra Señora de la Consolata. (2012) Proyecto Educativo Institucional. Institución Educativa Nuestra Señora de la Consolata. Cartagena.

Rodríguez, L; Carnota, R. (2015) Historia de las TIC en América Latina y el Caribe: Inicios, Desarrollos y Rupturas. Fundación telefónica. Madrid

Said-Hung, E, Iriarte Díaz-Granados,. Jabba, Barreto, Ballesteros, Vergara, E. Y Ordóñez, M. (2015) Fortalecimiento pedagógico en las universidades en Colombia a través de las TIC. Caso región Caribe. En: Educación XX1. Universidad del Norte. pág. 277-30

Serrano, A. (2003) Brecha digital: mitos y realidades. Ed Mexicali. 